\title{
Mechanisms of opioid tolerance: emerging evidence and therapeutic implications
}

\author{
Daisy T. Joo MD PhD CIP FRCP(C)
}

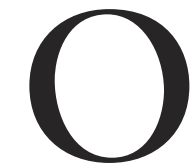
PIOIDS are still the mainstay therapy for treatment of a wide variety of acute and chronic pain disorders irrespective of their pathophysiology. In many instances, tolerance to opioid analgesia develops during the course of therapy and hampers the ability of the opioid to adequately relieve pain. Opioid tolerance is a "phenomenon" that is poorly understood and, thereby, difficult to remedy. Based on previous research, opioid rotation, N-methyl-D-aspartate (NMDA) receptor antagonism, and other adjunctive therapies have been tried with variable to limited benefit. However, research in the last few years has offered novel insights into opioid receptor G-protein associations, phosphorylation, trafficking, and interactions with other receptors that may change our understanding and treatment of opioid tolerance. This article explores the different cellular mechanisms which have been proposed to underlie opioid tolerance development and their possible therapeutic implications; concurrently, drawing attention to the complexities of the cellular machinery involved.

\section{G protein coupling}

Opioid receptors are G protein-coupled. Agonist binding to the opioid receptor initiates activity of its associated signalling $G$ protein and, in turn, other intracellular effector proteins in a cascade of activations resulting in the depression of neuronal activity that manifests as analgesia. There are three main subtypes of opioid receptors, namely $\mu, \delta$, and $\kappa$, which exist in pairs of receptors as homodimers or heterodimers. Associated signalling $G$ proteins also vary in subtypes depending on the composition of the dimeric opioid receptor unit. Analgesic properties of an opioid are due to the activation of homomeric $\mu$-opioid receptors (MORs) and associated $\mathrm{G}_{\mathrm{i} / \mathrm{o}}$ subtype of $\mathrm{G}$ proteins which is named for their inhibitory effect on adenylate cyclase and reduction of intracellular cAMP. However, in a few neurons, MOR associates with the $G_{s}$, adenylate cyclase and cAMP stimulatory, subtype of G protein which is of particular interest as its activation is related to a lack of analgesia and even pain. ${ }^{1}$

A switch in MOR association from $\mathrm{G}_{\mathrm{i} / \mathrm{o}}$ to $\mathrm{G}_{\mathrm{s}}$ proteins has been proposed to contribute to the development of opioid tolerance. Further evidence for $\mathrm{G}_{\mathrm{s}}$ protein participation in opioid tolerance is provided by the demonstration that inhibition of $G_{s}$ proteins through low dose naloxone "inhibition" of opioid receptors restores analgesia. ${ }^{2}$ Therefore, low dose naloxone may have a role in opposing opioid tolerance developed through an upregulated association of MORs with $\mathrm{G}_{\mathrm{s}}$ proteins. The mechanism responsible for a switch between $G_{i / o}$ to $G_{s}$ activation is unknown but may involve the dissociation of the MOR from its cognate $\mathrm{G}_{\mathrm{i} / \mathrm{o}}$ protein, resulting in receptor desensitization.

\section{G protein uncoupling}

Desensitization of the opioid receptor is a state in which relatively prolonged opioid binding reduces the function or impairs the ability of the receptor to signal through its intracellular pathway(s). Mu-opioid receptor desensitization which contributes to opioid tolerance development occurs with changes in the state of the receptor, its associated signalling proteins, and effector proteins. Although many mechanisms for MOR desensitization have been proposed, one involving the binding of an inhibitor protein is most intriguing. Following agonist exposure, MOR phosphorylation at Ser/Thr and Tyr residues causes conformational changes in the receptor. Phosphorylation of the MOR may be due to secondarily activated protein kinase $\mathrm{C}(\mathrm{PKC})$, protein kinase A activation, $\mathrm{Ca}^{2+}$-dependent calmodulin kinase, $\mathrm{G}$ protein-coupled receptor kinases, or mitogen-activated protein

CAN J ANESTH 2007 / 54: 12 / pp 969-976

From the Department of Anesthesia, Program in Neurosciences and Mental Health, Research Institute, Hospital for Sick Children, University of Toronto, Toronto, Ontario, Canada.

Address correspondence to: Dr. Daisy T. Joo, Department of Anesthesia, Hospital for Sick Children, 555 University Avenue, Room 5013C, Toronto, Ontario M5G 1X8, Canada. Phone: 416-813-5105 or 7543, ext. 1483; Fax: 416-813-7921; E-mail: daisy.joo@sickkids.ca 
kinases. ${ }^{3}$ Conformational changes in phosphorylated MORs allow intracellular proteins found ubiquitously in the central nervous system, called arrestins, to bind the receptor. ${ }^{4}$ Arrestin binding inhibits the interaction of the MOR with its signalling $\mathrm{G}_{\mathrm{i} / \mathrm{o}}$ protein. ${ }^{4}$ Mice genetically engineered to lack the $\beta$-arrestin 2 do not develop tolerance to morphine. ${ }^{5}$ Arrestin binding to opioid receptors occurs rapidly (< five minutes) and, therefore, may also account for acute opioid tolerance development.

Hence, the development of an opioid agonist that does not cause MORs to phosphorylate or bind arrestins, such as herkinorin, ${ }^{6}$ would resist tolerance development. This opioid is only in the early phases of drug discovery that may see the introduction of a new generation of tolerance-attenuating opioid therapeutics. Alternatively, another area for drug development may be in the identification of opioid adjuncts that prevent opioid tolerance by interfering with opioid receptor phosphorylation (i.e., through PKC inhibition) and arrestin binding.

However, arrestin binding has another effect on opioid tolerance in that it also recruits opioid receptors into endocytotic pathways and may actually reduce tolerance (see explanation below). ${ }^{7}$ More research into these seemingly conflicting roles of arrestins in opioid tolerance development is needed and may reveal selective actions of arrestin subtypes that can be therapeutically targeted. As well, specificity in the $G$ protein subtype, such as $G_{s}$, that is uncoupled by arrestin binding may be revealed to have implications on whether or not tolerance occurs by this mechanism.

\section{Receptor trafficking}

A major advancement in the understanding of opioid tolerance focused on the cellular mechanisms involved in G protein-coupled MOR trafficking. Historically, tolerance by the quantitative loss of receptor expression on the cell surface has been hypothesized but never adequately shown to occur for opioid receptors ${ }^{8}$ as it has for other $G$ protein-coupled receptors such as $\beta$-adrenergic or dopamine receptors. However, the number of binding sites for opioids has been demonstrated to decrease in opioid tolerance without a similar decline in the expression of the opioid receptor as quantified by mRNA levels. ${ }^{8}$ A plausible explanation for this came in 1996, when Keith et al. ${ }^{9}$ reported that different opioid agonists have varying abilities to cause MORs to desensitize and endocytose perhaps through the affinity of the agonist-receptor complex for binding arrestins. ${ }^{10}$ MORs sequestered by endocytosis through clathrin-coated pits are then targeted l) for dephosphorylation, re-sensitization, and recycling back to the cell surface ready to be activated again or 2) for degradation.

The re-sensitization and recycling of MORs is correlated to a reduced likelihood of tolerance development to opioids, such as sufentanil and methadone, which strongly promote the internalization of opioid receptors. ${ }^{11}$ In contrast, morphine activation does not cause MORs to internalize ${ }^{9}$ so tolerance to this opioid is more prevalent. This also accounts for decreased opioid binding sites without an effect on MOR surface expression in morphine tolerance. The differences between opioid agonists on their ability to internalize and recycle opioid receptors may account for some initial efficacy of opioid rotation strategies. However, interactions between agonists may also come into play as morphine in combination with an opioid that promotes endocytosis will cause morphine-bound MORs to uncharacteristically internalize, re-sensitize, and be recycled. This finding has prompted the suggestion that "cocktails" of opioids such as a low dose methadone combined with morphine used in therapy would attenuate tolerance development. ${ }^{12}$ In addition, methadone has inhibitory effects on NMDA receptors which can also prevent the development of opioid tolerance. ${ }^{13}$

\section{$\mathrm{N}$-methyl-D-aspartate receptor upregulation}

Multiple excitatory systems that contribute to reduced analgesia or increased pain have been shown to be secondarily activated or modulated by MOR activation. Inhibiting co-activated excitatory systems with adjuncts to opioid therapy have been proposed to reduce or attenuate opioid tolerance. Among the excitatory systems shown to be upregulated with MOR activity are orphanin receptors, ${ }^{14}$ cyclooxygenase,$^{15}$ calcitonin-gene related peptide, ${ }^{16}$ and substance P. ${ }^{16}$ The most attention has been focused on the enhancement of NMDA receptor function during opioid therapy. Although NMDA receptors are found ubiquitously in the central nervous system, they play a particularly important role in pain signalling via glutamatergic neurotransmission in the superficial laminae of the dorsal spinal cord. In dorsal horn neurons, NMDA receptors and MORs co-localize and participate in the transmission and modulation of pain signals to the brain. Activation of MORs enhances NMDA receptor function ${ }^{17}$ probably through an increase in PKC activity. ${ }^{18}$ Recent work in my laboratory reveals that acute and long-lasting NMDA response upregulation occurs upon remifentanil exposure in the same neurons that show enhanced NMDA current after prolonged incubation with morphine. ${ }^{19}$ This remifen- 
tanil-induced NMDA receptor enhancement requires the concurrent activity of both MORs and $\delta$-opioid receptors (DORs) (unpublished data).

Although the cellular processes contributing to and distinguishing between acute and chronic opioid tolerance by this mechanism are not clear, there is an abundance of evidence from animal studies that NMDA receptor inhibition during opioid exposure attenuates chronic opioid tolerance. ${ }^{13}$ A review of clinical literature on the effectiveness of ketamine in reducing opioid tolerance yields mixed results even though the adjuvant analgesia effects of this NMDA antagonist are well known..$^{20}$ Recently, more attention on the hyperalgesic effect of opioids and the development of opioid-induced hyperalgesia $(\mathrm{OIH})$ has focused on opioid receptor activated upregulation of NMDA receptor function as a possible mechanism. Clinically, it is difficult to distinguish between $\mathrm{OIH}$ and opioid tolerance. Therefore, it is likely that an increase in NMDA receptor function may contribute to both processes as to other pathological pain disorders, such as chronic and neuropathic pain. These lines of evidence on excitatory systems upregulated by opioid receptor activation provide the rationale for the clinical use of adjuvant treatments in opioid therapy such as with non-steroidal anti-inflammatory agents and NMDA antagonists. Further drug development may provide novel adjuncts to opioid therapy that target other enhanced excitatory systems.

\section{Mu-opioid receptor and delta-opioid receptor co- expression}

Although most of the focus has been on MORs thus far, it is also important to understand that interactions between the different opioid receptor subtypes occur, are complex, and change due to altered receptor expression during the establishment of opioid tolerance. For example, the activation of MORs by morphine increases the expression of DORs on cellular surfaces, ${ }^{21}$ thus increasing the probability for subtype interaction. The homomeric DOR activates a similar subset of $\mathrm{G}$ protein as the homomeric MOR. ${ }^{22}$ However, $\mu$ - $/ \delta$-opioid heterodimerization changes the agonist and antagonist binding properties of the opioid receptor complex and its associated $\mathrm{G}$ protein subtype (i.e., to $G_{z}$ ). ${ }^{23}$ This is thought to be due to a complex scaffolding of proteins to the homodimeric or heterodimeric opioid receptors which integrates its associated $\mathrm{G}$ proteins, phosphorylating proteins, and other effector proteins, ${ }^{24}$ possibly even PKC and NMDA receptors. The ligand selectivity may be based on a particular opioid's interaction with the receptor complex and its ability to stabilize or de-stabilize the structure within which enzymatic cascades are activated. Subsequently, the activated proteins govern the analgesic response and/or the development of tolerance. Therefore, different opioids will have different effects on intracellular processes that will alter their analgesic efficacy and side-effect profile. The association of DORs with MORs seems to increase the possibility for developing morphine tolerance, ${ }^{21}$ however, the reason for this is unclear. Also unclear is the effect of $G_{z}$ protein activation. Therefore, the interactions between the different subtypes of opioid receptor, in particular between MOR and DORs, need further examination such that selective inhibition, i.e., of the DOR or its trafficking, may be used to attenuate tolerance development.

\section{Conclusion}

A generalized overview on the research progress in areas of opioid receptor physiology and pathophysiology that may contribute to the development of opioid tolerance has been presented. Admittedly, there are problems in the translation of the cellular studies presented here to the whole organism. This is due, in part, to the expression systems used in many of the in vitro studies on opioid receptor desensitization and trafficking and, in part, to the complexities of the intracellular interactions involved. This complexity is compounded by pharmacogenetic variations in the opioid receptors expressed, anatomical differences of the opioid effects, and cell-specific cascades that determine the overall effect of an opioid on different tissues. However, it is this tissue differentiation that will narrow the search for the unifying opioid tolerance mechanism, if such an entity exists. On the assumption that there is a major pathway of activations through which a tolerance to opioids develops, the obvious anatomical locations would be along the pain transduction pathways. However, ascending pain transmission is also modulated by descending facilitation adding yet another facet of complexity.

Therefore, many challenges lie ahead in furthering our knowledge of opioid tolerance development. Despite major advances in opioid receptor physiology, researchers are only just starting to understand this very important system in the therapy of pain. Although the mechanism(s) underlying opioid tolerance is difficult to define, progress in basic science research is revealing possible targets for drug development of novel opioids or adjuvant treatments for clinical use to eliminate opioid tolerance. Until then, however, the problem of opioid tolerance will continue to plague the pain practitioner. 


\section{Acknowledgement}

My appreciation to Dr. Fiona Campbell for her comments regarding this manuscript.

\section{Mécanismes de} tolérance aux opiacés : nouvelles données et implications thérapeutiques

Les opiacés sont encore aujourd'hui la thérapie la plus courante dans le traitement d'une grande variété de troubles de la douleur aiguë et chronique et ce, sans considération aucune de leur physiopathologie. Dans de nombreux cas, une tolérance à l'analgésie à base d'opiacés se développe en cours de traitement et entrave la capacité de l'opiacé à soulager la douleur de façon efficace. La tolérance aux opiacés est un 'phénomène' mal compris et, par conséquent, difficile à gérer. Sur la base d'études antérieures, l'alternance de divers opiacés, l'antagonisme du récepteur $\mathrm{N}$-méthylD-aspartate (NMDA) et d'autres traitements adjuvants ont été testés, donnant des résultats variables à limités. Cependant, les recherches menées ces dernières années ont proposé des perspectives nouvelles sur les associations des récepteurs opiacés à la protéine $G$, à la phosphorylation, au trafic et aux interactions avec d'autres récepteurs. Ces données pourraient modifier notre compréhension et le traitement de la tolérance aux opiacés. Cet article explore les différents mécanismes cellulaires que l'on pense être à la base du développement d'une tolérance aux opiacés ainsi que leurs implications thérapeutiques possibles, tout en attirant l'attention sur la complexité des rouages cellulaires impliqués.

\section{Couplage aux protéines $\mathrm{G}$}

Les récepteurs opiacés sont couplés aux protéines $\mathrm{G}$. La liaison d'un agoniste au récepteur opiacé active la protéine de signalisation (protéine $\mathrm{G}$ ) associée, qui à son tour déclenche une cascade d'activations par d'autres protéines effectrices, avec pour résultat la chute de l'activité neuronale, laquelle se manifeste par l'analgésie. Il y a trois sous-types principaux de récepteurs opiacés, soit $\mu, \delta$, et $\kappa$, qui existent en paires de récepteurs en tant qu'homodimères ou qu'hétérodimères. Le sous-type des protéines $\mathrm{G}$ associées varie également selon la composition de l'unité du récepteur opiacé dimérique. Les propriétés analgésiques d'un opiacé proviennent de l'activation des récepteurs opiacés $\mu$ (MOR) homomériques et du sous-type $G_{i / o}$ des protéines $G$ associées, lesquelles sont nommées pour leur effet inhibiteur sur l'adénylcyclase et la réduction d'AMP cyclique (cAMP). Toutefois, dans certains neurones, les MOR s'associent au $\mathrm{G}_{\mathrm{s}}$, un stimulant de l'adénylcyclase et de cAMP - un sous-type de protéine G qui est particulièrement intéressant, étant donné que son activation est associée à une analgésie inexistante ou même à la douleur. ${ }^{1}$

On a proposé de remplacer l'association des MOR aux protéines $G_{i / o}$ par une association aux protéines $\mathrm{G}_{\mathrm{s}}$ afin d'inhiber le développement de la tolérance aux opiacés. D'autres preuves du rôle que joue la protéine $\mathrm{G}_{\mathrm{s}}$ dans la tolérance aux opiacés ont été apportées en démontrant que l'inhibition des protéines $G_{s}$ par une «inhibition» des récepteurs opiacés de la naloxone à faible dose restaure l'analgésie. ${ }^{2}$ Ainsi, la naloxone à faible dose pourrait jouer un rôle en contrecarrant la tolérance aux opiacés développée par une association régulée à la hausse des MOR aux protéines $\mathrm{G}_{\mathrm{s}}$. Le mécanisme sur lequel se fonde le remplacement entre l'activation de la protéine $\mathrm{G}_{\mathrm{i} / \mathrm{o}}$ et l'activation de la protéine $\mathrm{G}_{\mathrm{s}}$ est inconnu, mais il pourrait impliquer la dissociation des MOR de leur protéine apparentée $\mathrm{G}_{\mathrm{i} / \mathrm{o}}$, ce qui aurait pour résultat la désensibilisation des récepteurs.

\section{Découplage des protéines $\mathrm{G}$}

La désensibilisation du récepteur opiacé est un état durant lequel une liaison opiacée relativement prolongée réduit la fonction et entrave la capacité du récepteur à émettre des signaux par sa (ses) voie(s) intracellulaire(s). La désensibilisation du récepteur opiacé mu $(\mu)$, qui stimule le développement d'une tolérance aux opiacés, a lieu lors de changements d'état du récepteur, de ses protéines de signalisation associées, et des protéines effectrices. Bien que de nombreux mécanismes de la désensibilisation des MOR aient été suggérés, l'un d'eux, impliquant la liaison d'une protéine inhibitrice, est intrigant. Après une exposition aux agonistes, la phosphorylation des MOR aux résidus de sérine/thréonine et de tyrosine provoque des changements de conformation du récepteur. La phosphorylation des MOR pourrait avoir pour cause la protéine kinase $\mathrm{C}$ (PKC) activée secondairement, la calmoduline kinase $\mathrm{Ca}^{2+}$ dépendante, les récepteurs kinases liés aux protéines $\mathrm{G}$ ou les protéines kinases activées par des agents mitogènes. ${ }^{3}$ Les transforma- 
tions de conformation visibles dans les MOR ayant subi une phosphorylation permettent à des protéines intracellulaires omniprésentes dans le système nerveux central, appelées arrestines, de se lier au récepteur. ${ }^{4}$ La liaison aux arrestines inhibe l'interaction entre les MOR et leur protéine de signalisation $\mathrm{G}_{\mathrm{i} / \mathrm{o}}{ }^{4}$ Les souris génétiquement modifiées pour ne pas avoir de $\beta$-arrestine 2 ne développent pas de tolérance à la morphine. ${ }^{5}$ La liaison de l'arrestine aux récepteurs opiacés est rapide (< cinq minutes) et, de ce fait, pourrait également expliquer le développement d'une tolérance aiguë aux opiacés.

Dès lors, le développement d'un agoniste opiacé ne produisant pas de phosphorylation des MOR ou de liaison aux arrestines, tel que l'herkinorine ${ }^{6}{ }^{6}$ résisterait au développement d'une tolérance. Cet opiacé n'est actuellement que dans les phases précoces de développement, mais il pourrait signifier l'introduction d'une nouvelle génération de traitements opiacés atténuant la tolérance. Un autre domaine possible de développement de médicament pourrait avoir lieu dans le champ de l'identification des adjuvants aux opiacés qui empêchent la tolérance aux opiacés en agissant sur la phosphorylation des récepteurs (c.-à-d. par l'inhibition de la $\mathrm{PKC}$ ) et la liaison à l'arrestine.

Cependant, la liaison à l'arrestine a également un autre effet sur la tolérance aux opiacés, en ce qu'elle attire aussi des récepteurs opiacés vers les voies endocytosiques, réduisant ainsi la tolérance (voir explication ci-dessous). ${ }^{7}$ Des recherches supplémentaires quant aux rôles apparemment contradictoires des arrestines dans le développement de la tolérance aux opiacés sont nécessaires et pourraient révéler des actions sélectives de sous-types d'arrestine qui pourraient être ciblées par des traitements. En outre, la spécificité du sous-type de protéine $G$, telle que la protéine $G_{s}$, qui n'est pas associée à une liaison à l'arrestine, pourrait avoir des implications sur la tolérance ou au contraire la non-tolérance liée à ce mécanisme.

\section{Trafic des récepteurs}

Un des progrès majeurs dans la compréhension de la tolérance aux opiacés touche aux mécanismes cellulaires sur lesquels sont fondés le trafic des MOR liés aux protéines $\mathrm{G}$. Traditionnellement, on a expliqué la tolérance par une perte quantitative d'expression des récepteurs sur la surface cellulaire a été suggérée ; cependant, cette hypothèse n'a jamais été démontrée pour les récepteurs opiacés, ${ }^{8}$ tel que cela a été le cas pour d'autres récepteurs liés aux protéines $G$, comme les récepteurs $\beta$-adrénergiques ou dopaminergiques. Toutefois, il a été démontré que le nombre de sites de liaison pour les agents opiacés diminuaient lors d'une tolérance aux opiacés et ce, sans déclin similaire dans l'expression du récepteur opiacé telle que quantifiée par les niveaux d'ARNm. ${ }^{8}$ Une explication possible de ce phénomène a été émise en 1996, lorsque Keith et coll. ${ }^{9}$ ont démontré que différents agonistes opiacés possèdent des capacités variables à provoquer une désensibilisation et une endocytose des MOR, peut-être par le biais de l'affinité du complexe agoniste-récepteur pour les arrestines liantes. ${ }^{10}$ Les MOR séquestrés par endocytose via des puits couverts de clathrine sont ensuite ciblés 1) pour la déphosphorylation, la resensibilisation et une récupération jusqu'à la surface cellulaire, où ils sont prêts à être réactivés ou 2) pour la dégradation.

La resensibilisation et le recyclage des MOR sont associés à une probabilité réduite de développement de tolérance aux opiacés tels le sufentanil ou la méthadone, qui encouragent fortement l'intériorisation des récepteurs opiacés. ${ }^{11}$ En revanche, l'activation par la morphine ne produit pas une intériorisation des MOR ;9 ainsi, la tolérance à cet opiacé est plus fréquente. Ceci a également pour conséquence un nombre réduit de sites de liaison aux opiacés, sans avoir un effet sur l'expression de surface des MOR dans la tolérance à la morphine. Les différences entre les agonistes opiacés quant à leur capacité à intérioriser et recycler les récepteurs opiacés pourraient être à la base de l'efficacité première des stratégies de rotation des opiacés. Toutefois, les interactions entre les agonistes pourraient également jouer un rôle, étant donné que la morphine combinée à un opiacé qui stimule l'endocytose aura pour résultat que les MOR liés à la morphine soient intériorisées, resensibilisés et recyclés, ce qui n'est pas typique. Cette découverte a donné naissance à la proposition que des « cocktails » d'opiacés, comme par exemple de la méthadone à faible dose associée à de la morphine dans un traitement, atténueraient le développement d'une tolérance. ${ }^{12}$ En outre, la méthadone a des effets inhibiteurs sur les récepteurs NMDA, ce qui peut également prémunir contre le développement d'une tolérance aux opiacés. ${ }^{13}$

\section{Régulation à la hausse du récepteur N-méthyl-D- aspartate}

Il a été démontré que de nombreux systèmes excitateurs qui contribuent à une analgésie réduite ou à une douleur plus forte sont secondairement activés ou modulés par l'activation des MOR. On a proposé d'inhiber les systèmes excitateurs co-activés avec des adjuvants au traitement opiacé afin de réduire ou d'atténuer la tolérance aux opiacés. Parmi les systèmes excitateurs qui sont régulés à la hausse avec une activité des MOR, il y a : les récepteurs d'orphanine, ${ }^{14}$ la 
cyclo-oxygénase, ${ }^{15}$ le peptide lié au gène de la calcitonine, ${ }^{16}$ et la substance P. ${ }^{16}$ L'attention des chercheurs s'est surtout portée sur l'amélioration de la fonction des récepteurs NMDA pendant le traitement aux opiacés. Bien que les récepteurs NMDA soient omniprésents dans le système nerveux central, ils jouent un rôle particulièrement important dans la signalisation de la douleur via une neurotransmission glutamatergique dans les lames superficielles de la moelle épinière dorsale. Dans les neurones de la corne dorsale, les récepteurs NMDA et les MOR sont tous deux présents et participent à la transmission et à la modulation des signaux de douleur au cerveau. L'activation des MOR stimule la fonction des récepteurs $\mathrm{NMDA}^{17}$ probablement par une augmentation de l'activité de la PKC. ${ }^{18}$ Des recherches récentes effectuées dans mon laboratoire ont révélé que la régulation à la hausse d'une réaction prolongée et aiguë des NMDA survient suite à l'exposition au rémifentanil dans les neurones qui montrent un courant de NMDA augmenté après une incubation prolongée avec de la morphine. ${ }^{19}$ Cette stimulation accrue des récepteurs NMDA provoquée par le rémifentanil nécessite l'activité simultanée des MOR et des récepteurs $\delta$-opiacés (DOR) (résultats non publiés).

Bien que les processus cellulaires contribuant à la tolérance aux opiacés et provoquant une distinction entre la forme aiguë et chronique par ce mécanisme ne soient pas clairs, il existe une multitude de preuves provenant d'études animales que l'inhibition des récepteurs NMDA pendant une exposition aux opiacés réduit la tolérance chronique aux opiacés. ${ }^{13}$ Une revue de la littérature clinique traitant de l'efficacité de la kétamine pour réduire une tolérance aux opiacés donne des résultats mitigés, malgré le fait que les effets adjuvants sur l'analgésie de cet antagoniste NMDA soient bien connus. ${ }^{20}$ Récemment, les recherches portant sur l'effet hyperalgésique des opiacés et le développement d'une hyperalgésie provoquée par les opiacés $(\mathrm{OIH})$ se sont davantage concentrées sur la régulation à la hausse de la fonction des récepteurs NMDA activée par les récepteurs opiacés en tant que mécanisme possible. Il est cliniquement difficile de faire une distinction entre l'OIH et la tolérance aux opiacés. C'est pourquoi il est possible qu'une fonction des récepteurs NMDA accrue puisse contribuer aux deux processus ainsi qu'à d'autres troubles pathologiques comme la douleur chronique et la douleur neuropathique. Ces pistes de preuves concernant les systèmes excitateurs régulés à la hausse par l'activation des récepteurs opiacés fournissent une justification pour l'utilisation clinique de traitements adjuvants lors d'une thérapie à base d'opiacés, comme par exemple avec des agents anti-inflammatoires non stéroïdiens et des antagonistes NMDA. Des médicaments en développement pourraient fournir de nouveaux adjuvants au traitement par opiacés qui visent d'autres systèmes excitateurs stimulés.

\section{Expression conjointe du récepteur mu-opiacé et delta-opiacé}

Bien que nous ayons principalement porté notre attention sur les MOR jusqu'à présent, il est important de comprendre que des interactions entre les différents sous-types de récepteurs opiacés surviennent, sont complexes, et se modifient à cause d'une expression des récepteurs modifiée pendant la mise en place d'une tolérance aux opiacés. Par exemple, l'activation des MOR par la morphine augmente l'expression des DOR sur les surfaces cellulaires ${ }^{21}$ augmentant ainsi la probabilité d'une interaction entre les sous-types. Le DOR homomérique active un sous-ensemble similaire de protéines $\mathrm{G}$ que le MOR homomérique. ${ }^{22}$ Toutefois, l'hétérodimérisation $\mu-/ \delta$ - des récepteurs opiacés modifie les propriétés de liaison de l'agoniste et de l'antagoniste du complexe du récepteur opiacé et de son sous-type associé de protéine $\mathrm{G}$ (c.-à-d. jusqu'à $\left.G_{z}\right) \cdot{ }^{23}$ On pense que cela est dû à un échafaudage complexe de protéines sur les récepteurs opiacés homodimériques et hétérodimériques, lequel intègre ses protéines $\mathrm{G}$ associées, ses protéines de phosphorylation et d'autres protéines effectrices, ${ }^{24}$ et même peutêtre la PKC et les récepteurs NMDA. La sélectivité du ligand pourrait être fondée sur l'interaction d'un opiacé en particulier avec le complexe des récepteurs et sa capacité à stabiliser ou au contraire déstabiliser la structure dans laquelle les cascades enzymatiques sont activées. Par la suite, les protéines activées régissent l'effet analgésique et/ou le développement d'une tolérance. C'est pourquoi différents opiacés auront différents effets sur les processus intracellulaires qui modifieront leur efficacité analgésique et leur profil d'effets secondaires. L'association des DOR aux MOR semble augmenter la possibilité de développer une tolérance à la morphine, ${ }^{21}$ mais nous ne savons pas pourquoi. Nous ne connaissons pas non plus l'effet de l'activation de la protéine $\mathrm{G}_{\mathrm{z}}$. C'est pourquoi les interactions entre les différents sous-types de récepteurs opiacés, et en particulier entre les MOR et les DOR, doivent faire l'objet de recherches plus approfondies afin que l'inhibition sélective, c.-à-d. du DOR ou de son trafic, puisse être utilisée pour atténuer le développement d'une tolérance.

\section{Conclusion}

Nous avons présenté un tour d'horizon généralisé des 
progrès de la recherche dans les domaines de la physiologie et de la physiopathologie des récepteurs opiacés qui pourraient être à la base du développement d'une tolérance aux opiacés. Il faut reconnaître qu'il existe des problèmes dans la traduction des études cellulaires présentées ici à l'organisme tout entier. Ceci est d'une part dû aux systèmes d'expression utilisés dans beaucoup d'études in vitro portant sur la désensibilisation et le trafic des récepteurs opiacés et, d'autre part, à la complexité des interactions intracellulaires en question. Cette complexité est amplifiée par les variations pharmacogénétiques des récepteurs opiacés exprimés, les différences anatomiques des effets des opiacés, et des cascades spécifiques aux cellules qui définissent l'effet global d'un opiacé sur différents tissus. Cependant, c'est précisément cette différentiation tissulaire qui permettra de limiter le champ de recherche pour le mécanisme unifiant de tolérance aux opiacés, si un tel mécanisme existe. Partant de l'hypothèse qu'il y a une importante voie d'activations par laquelle une tolérance aux opiacés se développe, les emplacements anatomiques évidents se situeraient le long des voies de transduction de la douleur. Toutefois, la transmission ascendante de la douleur est aussi modulée par la facilitation descendante, ajoutant ainsi une facette supplémentaire à la complexité de ce mécanisme.

Ainsi, de nombreux défis nous attendent avant de pouvoir approfondir nos connaissances du développement de la tolérance aux opiacés. En dépit d'importants progrès dans la connaissance de la physiologie des récepteurs opiacés, les chercheurs commencent tout juste à comprendre ce système essentiel dans le traitement de la douleur. Bien que le(s) mécanisme(s) sousjacent(s) à la tolérance aux opiacés soi(en)t difficiles à déterminer, les progrès dans la recherche scientifique fondamentale révèlent des cibles possibles pour le développement d'opiacés nouveaux ou de traitements adjuvants à utiliser dans le milieu clinique pour éliminer la tolérance aux opiacés. Jusqu'à ce jour, toutefois, le problème de la tolérance aux opiacés ne cessera de tourmenter le spécialiste de la douleur.

\section{References}

1 Gintzler AR, Chakrabarti S. Post-opioid receptor adaptations to chronic morphine: altered functionality and associations of signaling molecules. Life Sci 2006; 79: 717-22.

2 Crain SM, Shen KF. Antagonists of excitatory opioid receptor functions enhance morphine's analgesic potency and attenuate opioid tolerance/dependence liability. Pain 2000; 84: 121-31.

3 Lin JG, Anand KJ. Protein kinases modulate the cellular adaptations associated with opioid tolerance and dependence. Brain Res Brain Res Rev 2001; 38: 1-19.

4 Johnson EE, Christie MJ, Connor M. The role of opioid receptor phosphorylation and trafficking in adaptations to persistent opioid treatment. Neurosignals 2005; 14 : 290-302.

5 Bohn LM, Dykstra LA, Lefkowitz RJ, Caron MG, Barak $L S$. Relative opioid efficacy is determined by the complements of the G protein-coupled receptor desensitization machinery. Mol Pharmacol 2004; 66: 106-12.

6 Groer CE, Tidgewell K, Moyer RA, et al. An opioid agonist that does not induce micro-opioid receptor-arrestin interactions or receptor internalization. Mol Pharmacol 2007; 71: 549-57.

7 Finn AK, Whistler JL. Endocytosis of the mu opioid receptor reduces tolerance and a cellular hallmark of opiate withdrawal. Neuron 2001; 32: 829-39.

8 Marie N, Aguila B, Allouche S. Tracking the opioid receptors on the way of desensitization. Cell Signal 2006; 18: 1815-33.

9 Keith DE, Murray SR, Zaki PA, et al. Morphine activates opioid receptors without causing their rapid internalization. J Biol Chem 1996; 271: 19021-4.

$10 Z$ Zn $Z$. The role of opioid receptor internalization and beta-arrestins in the development of opioid tolerance. Anesth Analg 2005; 101: 728-34.

11 Koch T, Widera A, Bartzsch K, et al. Receptor endocytosis counteracts the development of opioid tolerance. Mol Pharmacol 2005; 67: 280-7.

$12 \mathrm{He}$ L, Whistler JL. An opiate cocktail that reduces morphine tolerance and dependence. Curr Biol 2005; 15 : 1028-33.

13 Trujillo $K A$. Are NMDA receptors involved in opiateinduced neural and behavioral plasticity? A review of preclinical studies. Psychopharmacology (Berl) 2000; 151: 121-41.

14 Ueda $H$. Locus-specific involvement of anti-opioid systems in morphine tolerance and dependence. Ann N Y Acad Sci 2004; 1025: 376-82.

15 Hsu MM, Wong CS. The roles of pain facilitatory systems in opioid tolerance. Acta Anaesthesiol Sin 2000; 38: 155-66.

16 Trang T, Quirion R, Jhamandas K. The spinal basis of opioid tolerance and physical dependence: involvement of calcitonin gene-related peptide, substance $P$, and arachidonic acid-derived metabolites. Peptides 2005; 26: 1346-55.

17 Chen L, Huang LY. Sustained potentiation of NMDA receptor-mediated glutamate responses through activation of protein kinase C by a mu opioid. Neuron 1991; 7: 319-26.

18 Chen L, Huang LY. Protein kinase C reduces $\mathrm{Mg} 2+$ block of NMDA-receptor channels as a mechanism of modulation. Nature 1992; 356: 521-3. 
19 Zhao M, Joo DT. Subpopulation of dorsal horn neurons displays enhanced N-methyl-D-aspartate receptor function after chronic morphine exposure. Anesthesiology 2006; 104: 815-25.

20 Subramaniam K, Subramaniam B, Steinbrook RA. Ketamine as adjuvant analgesic to opioids: a quantitative and qualitative systematic review. Anesth Analg 2004; 99: 482-95.

21 Zhang X, Bao L, Guan JS. Role of delivery and trafficking of delta-opioid peptide receptors in opioid analgesia and tolerance. Trends Pharmacol Sci 2006; 27: 324-9.

22 Alt A, Clark MJ, Woods JH, Traynor JR. Mu and delta opioid receptors activate the same G proteins in human neuroblastoma SH-SY5Y cells. Br J Pharmacol 2002; 135: 217-25.

23 Fan T, Varghese G, Nguyen T, Tse R, O'Dowd BF, George SR. A role for the distal carboxyl tails in generating the novel pharmacology and $\mathrm{G}$ protein activation profile of $\mathrm{mu}$ and delta opioid receptor hetero-oligomers. J Biol Chem 2005; 280: 38478-88.

24 Milligan $G$. Opioid receptors and their interacting proteins. Neuromolecular Med 2005; 7: 51-9. 